the stability of short lengths of helix, which are often observed, may sometimes involve this mechanism, and probably in general requires the non-aqueous environment provided by clustered non-polar side chains.

\section{Progress against Plant Disease}

Producing plants which are resistant to disease is one of the largest problems facing plant breeders and, to judge from the annual report, it seems to be one that the Plant Breeding Institute in Cambridge is tackling with some success. The report (price 7s. 6d.) describes the latest progress of the institute's breeding programme, which is aimed at producing varieties showing disease resistance combined with the essential improved yield and quality in cereals, potatoes, brassicas, sugar beet and forage crops.

The cytogenetics department is particularly proud of a breeding variety of wheat called 'Compair' which has been developed from crosses between hexaploid wheat, Triticum aestivum, a wild Aegean species, Aegilops comosa, and another species, Aegilops speltoides. By exploiting single chromosomes, which exercise control over the behaviour of the whole chromosome complement during meiosis, it has been possible to integrate into the chromosome complement of $T$. aestivum genes, originally from $A$. comosa, which confer resistance to yellow rust disease. This variety, 'Compair', is to be used by breeders as parent mater- ial in producing new disease resistant varieties - Compair' itself shows a low yield, and so it is not suitable as a crop variety. Another cytogenetic success has been the introduction of a chromosome which confers resistance to both yellow rust and mildew from rye into wheat.

A new development by the cereals section has been the production of spring wheats which have a high degree of tolerance to the mineral deficiencies of fen soils. Further crosses are being carried out to introduce disease resistance and improve baking quality. A very important variety of winter wheat which shows a much higher yield than previous varieties is 'Maris Ranger'. This variety is also resistant to all races of yellow rust known in Britain. It has now completed the trials of the National Institute of Agricultural Botany and has been placed on the recommended list.

Work has been in progress at the Plant Breeding Institute for some years on semi-dwarf wheats originating from a Japanese variety called 'Norin 10'. These plants have shorter internodes than usual, but no change in the number of internodes, so that there is the opportunity for producing a lot of grain with less vegetative growth than usual. The chief drawback to the use of these varieties in Britain has been their extreme susceptibility to yellow rust and powdery mildew, but crosses with other disease resistant wheats have produced some very short strawed disease resistant lines. Work is continuing with material which shows considerable promise.

\title{
Why do Fish School?
}

by

D. H. CUSHING

F. R. HARDEN JONES

Fisheries Laboratory,

Lowestoft
Studies of fish behaviour suggest that the habit of schooling, whatever its mechanism, is an advantage to potential prey.
The survival value of the habit of fish schooling is the subject of a recent article by Dr C. M. Breder ${ }^{1}$, who has written a great deal about the social aspects of schooling. $\mathrm{He}$ has worked in streams, lakes and hatcheries in fresh water and in the sea off Bimini and off the west coast of Florida. His photographs of fish schools are brilliant ${ }^{2}$, but they were taken in shallow or inshore well-lit waters. As fisheries biologists, we work on this problem in the open sea or in the deep ocean with acoustic equipment.

Breder" defines a school as "a large number of fish swimming together" all orientated in the same direction, and considers that "the group is permanent except ... on extraordinarily dark nights"-permanent because it is a common observation that shoaling fish are all of the same length. But echo sounder records show that schooling fish tend to disperse into masses of individuals at night and to gather again into schools in daytime. Magnuson and Prescott ${ }^{3}$ have suggested that the leaning behaviour of tuna in the early dawn allows them to use their silvery sides (Denton and $\mathrm{Nicol}^{4}$ ) to aggregate from extreme visual ranges. Because fish cruise at three lengths a second, a short period of activity in the early morning should segregate the fish into schools of specific sizes quite readily. Consequently, it seems unlikely that schools are permanent or need to be permanent. Spawning or pre-spawning schools may endure for longer than a day, because they do sometimes retain their identity at night (herring ${ }^{5,8}, \operatorname{cod}^{7}$ ).

An earlier analysis by Breder $^{8}$ treats a fish school as being held together by attractive forces and repulsive forces, by analogy with centrifugal and centripetal forces. but the forces are not defined. The mathematical model was not fully developed and the survival value of schooling remained a subject for speculation.

There are now three articles which present models of schooling behaviour. The first, by Brock and Riffenburgh', emphasizes the importance of schooling as a factor in reducing predation. These authors define two probabilities-the first that of a single prey being sighted by a predator, and the second that of a predator sighting a school. The expectation of the number of prey being eaten depends on the number of predators, the number of prey and the distance at which the predator sights the prey. The expectation for prey randomly distributed is $E_{1}$ and that for schooled prey is $E_{2}$; when schooling is of advantage to the prey, $E_{2}<E_{1}$. This treatment has the advantage of being independent of time and of prey density. Furthermore, the expectations can be readily expressed as simple volumes.

Let us assume that there are $r^{1} / c$ prey along the radius $\left(r^{1}\right)$ of a spherical school. Then the number of prey 